\title{
Not just BLAST nt: WGS database joins the party
}

Running title:

\section{draftGenomes: Beyond BLAST nt}

\author{
Jose Manuel Martíi ${ }^{1,2,(-}$ and Carlos P. Garay ${ }^{1,2,(-)}$ \\ ${ }^{1}$ Institute for Integrative Systems Biology (ISysBio), Valencia, Spain. \\ ${ }^{2}$ Canfranc Underground Laboratory (LSC), Huesca, Spain.
}

May 28, 2019

\begin{abstract}
Since its introduction in 1990 and with over 50k citations, the NCBI BLAST family has been an essential tool of in silico molecular biology. The BLAST nt database, based on the traditional divisions of GenBank, has been the default and most comprehensive database for nucleotide BLAST searches and for taxonomic classification software in metagenomics. Here we argue that this is no longer the case. Currently, the NCBI WGS database contains one billion reads (almost five times more than GenBank), and with 4.4 trillion nucleotides, WGS has about 14 times more nucleotides than GenBank. This ratio is growing with time. We advocate a change in the database paradigm in taxonomic classification by systematically combining the nt and WGS databases in order to boost taxonomic classifiers sensitivity. We present here

Correspondence: jose.m.marti@uv.es and cpenyaalsc-canfranc.es
\end{abstract}


a case in which, by adding WGS data, we obtained over five times more classified reads and with a higher confidence score. To facilitate the adoption of this approach, we provide the draftGenomes script.

\section{Author summary}

Culture-independent methods are revolutionizing biology. The NIH/NCBI Basic Local Alignment Search Tool (BLAST) is one of the most widely used methods in computational biology. The BLAST nt database has become a de facto standard for taxonomic classifiers in metagenomics. We believe that it is time for a change in the database paradigm for such a classification. We advocate the systematic combination of the BLAST nt database with genomes of the massive NCBI Whole-Genome Shotgun (WGS) database. We make draftGenomes available, a script that eases the adoption of this approach. Current developments and technologies make it feasible now. Our recent results in several metagenomic projects indicate that this strategy boosts the sensitivity in taxonomic classifications.

Keywords - metagenomics, taxonomic classification, draft genomes, whole genome, NCBI, GenBank, WGS, sensitivity

\section{Introduction}

The NIH (National Institute of Health) created the NCBI (National Center for Biotechnology Information) in 1988 to advance information systems devoted to molecular biology. The GenBank nucleic acid sequence database was one of its first hosted projects (Benson et al. 2013). When the NCBI Taxonomy database project was launched in 1991, GenBank was moved under its umbrella (Federhen 2011). Nowadays, NCBI provides computational resources and data retrieval systems for the study of several other sets of biological data arranged in various additional databases (NCBI Resource Coordinators 2013). The NCBI BLAST (Basic Local Alignment Search Tool) software (Altschul et al. 1990) uses a standard set of BLAST databases for protein, nucleotide, and translated BLAST searches. NCBI frequently releases pre-formatted versions of these databases as compressed files, which any user can download from the NCBI BLAST FTP site. The NCBI BLAST $n t$ database is one of those databases and is actually the default for nucleotide BLAST searches (NCBI Resource Coordinators 2013). 
Common misunderstandings about the BLAST nt database include the belief that it is a strictly nonredundant nucleotide sequence database, and that all sequences from the NCBI nucleotide databases are included. Currently (as of May 2019), it contains "partially non-redundant nucleotide sequences from all traditional divisions of GenBank, EMBL, and DDBJ; excluding GSS, STS, PAT, EST, HTG, and WGS" (NCBI 2017). Therefore, while $\mathrm{nr}$ is strictly non-redundant, $n t$ is partially non-redundant (NCBI 2017).

Concretely, the BLAST nt database includes the NCBI nucleotide sequences databases listed in Table $\underline{1}$ but does not include the NCBI nucleotide sequences databases indexed in Table 2, which also details how to obtain those resources downloadable from NCBI.

Table 1. NCBI nucleotide sequences databases included in BLAST nt database.

\begin{tabular}{cl}
\hline NCBI database & Contents \\
\hline TSA & Transcriptome shotgun data \\
ENV & Environmental samples \\
PHG & Phages \\
BCT & Bacteria \\
INV & Invertebrates \\
VRL & Viruses \\
MAM & Other mammals \\
PLN & Plants \\
SYN & Synthetic \\
VRT & Other vertebrates \\
UNA & Unannotated \\
PRI & Primates \\
ROD & Rodents \\
HTC & High-throughput cDNA \\
\hline
\end{tabular}

Notice that the nt database includes genomes sequenced by a whole genome shotgun strategy but not the WGS database, a massive database from NCBI (Wheeler et al. 2007) that contains incomplete genomes sequenced by whole genome shotgun. These nucleotide sequences belong to hundreds of thousands of different sequencing projects which should be located and downloaded individually. Precisely, draftGenomes understands this project-basis approach and automates the collection of all the sequences in the NCBI WGS database belonging to a taxonomic subtree. 
Table 2. NCBI nucleotide sequences databases NOT included in BLAST nt database.

\begin{tabular}{lll}
\hline NCBI DB & Contents & $\begin{array}{l}\text { Additional files to download } \\
\text { from NCBI FTP servers }\end{array}$ \\
\hline GSS & Genome survey sequences & gss.*tar.gz \\
\hline STS & Sequence tagged sites & sts.gz \\
\hline PAT & Patented sequences & patnt.gz \\
\hline EST & Expressed sequence tags & est_human.gz, \\
& & est_others.gz \\
\hline HTC & High-throughput cDNA & htgs.*tar.gz \\
\hline WGS & Whole-genome shotgun data & Use draftGenomes \\
\hline
\end{tabular}

Strictly speaking, the WGS database is a division of the GenBank database that appeared in April 2002, 21 years after the first release of GenBank. Nevertheless, the name 'GenBank' is often used when referring to the traditional GenBank divisions, which do not include the WGS database. We will keep this tacit agreement throughout this manuscript and will consider GenBank and WGS as independent databases.

In August 2005, after only 3 years and four months in use, the WGS database surpassed GenBank in number of nucleotides. Years later, in June 2014, the release 202 of the WGS database exceeded GenBank also in the number of sequences included. In recent years, the number of bases and sequences in the WGS database is roughly doubling every two years.

Figure 1 shows the evolution of both the number of bases and the number of sequences (in semilogarithmic scale) contained in the GenBank and WGS databases, from December 1982 to April 2019. Interestingly, the GenBank database presented an exponential growth in the early stages, very similar to the current increase rate of the WGS database. In recent years, the growth of the GenBank database has slowed down significantly. The number of GenBank bases increased threefold in the last decade, far from the 48 -fold increase that took place from 1998 to 2008. 


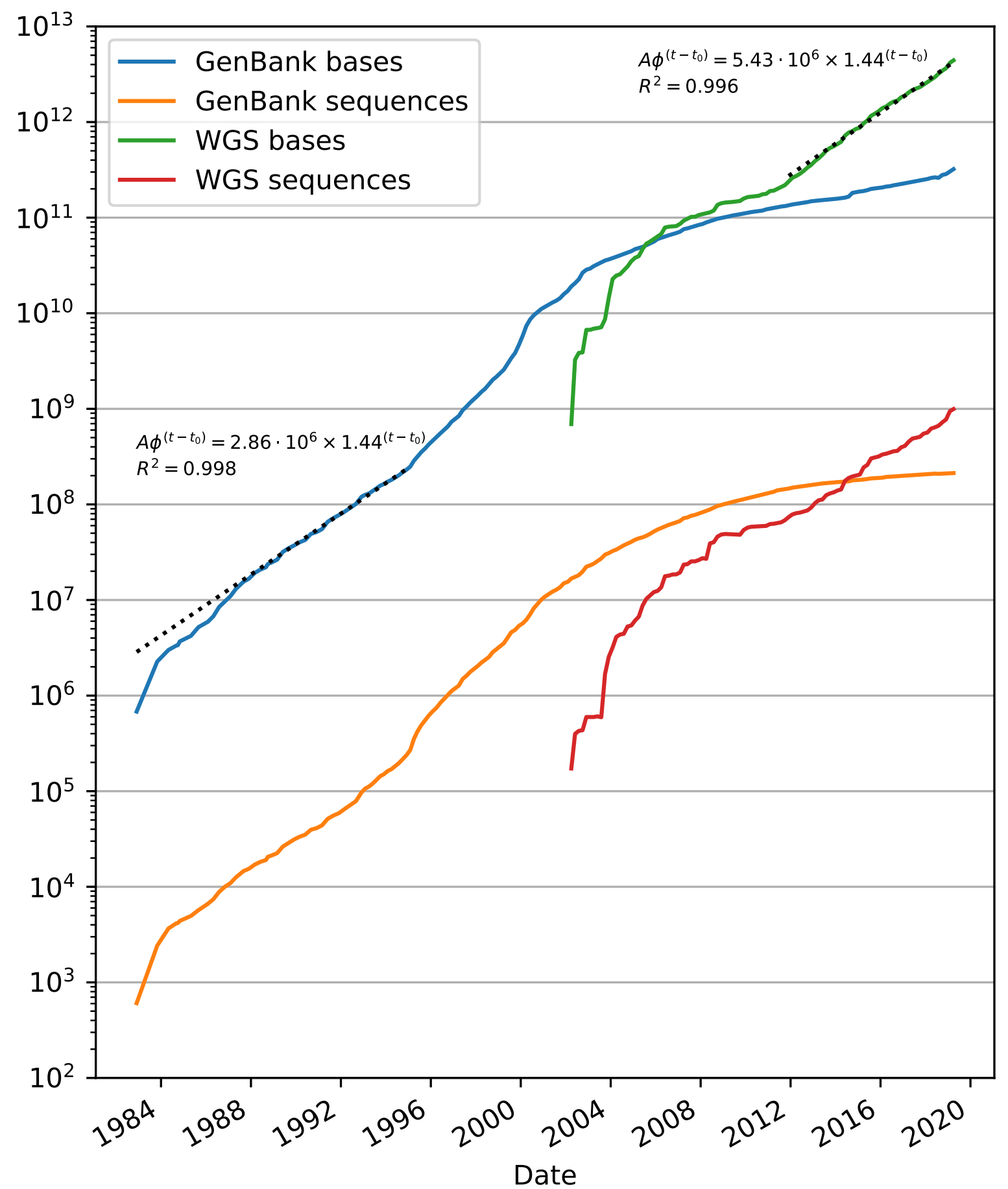

Figure 1. Temporal evolution of the NCBI GenBank and WGS databases from December 1982 to April 2019. The $y$-axis indicates the number of bases and sequences (in logarithmic scale) and the $\mathrm{x}$-axis the time in years (with December 1982 as the initial time $t_{0}$ ). Additionally, the figure shows as dotted black lines the fits of exponential models to the number of bases. Interestingly, from the beginning to December 1994, GenBank shows an exponential growth rate in the number of bases very similar to that of WGS from December 2011 to the last release (April 2019). 


\section{Design and implementation}

\subsection{Overview}

The draftGenomes software greatly simplifies the otherwise arduous task of collecting all the NCBI WGS sequences related to a NCBI taxonomic identifier (TaxID) at any taxonomic level. This script downloads the appropriate sequence files from NCBI WGS projects and parses them to generate a single coherent fasta file by parsing the sequence headers and updating them if needed.

\subsection{Details}

Depending on the chosen TaxID, the download and analysis of the NCBI WGS projects can take a long time (and require a lot of disk space). To deal with such a common situation, the script shows progress indicators and automatically recovers from several errors. Furthermore, draftGenomes has been fitted with a resume mode in case of any fatal interruption of the process.

We describe below further modes of operation:

- The reverse mode enables another instance of the script to manage the download of sequences in reverse order without interfering with the first one, which is also parsing the sequences to generate the resulting fasta file.

- The force mode ignores previous downloads and recreates the final FASTA file in spite of previous runs of the script for the same TaxID.

- The download mode is used to download without parsing the WGS project files.

- The verbose mode substitutes the progress indicator with details about every project parsed.

The draftGenomes code has been tested successfully in $\sim$ TB downloads from the NCBI servers with several forced and unforced interruptions.

\subsection{Installing draftGenomes}

draftGenomes is a compact script that can be installed just cloning the GitHub repository, downloading the script, or even pasting the source code using any text editor. 


\subsection{Running draftGenomes}

draftGenomes just requires a Python 3 interpreter. No other packages beyond the Python Standard Library ones are needed. Running ./draftGenomes.py --help will print all the possibilities and details of the draftGenomes software. The output files have the format: WGS4taxid\{include\}-\{exclude\}.fa where $\{$ include\} is the TaxID of the root of the taxonomic subtree of interest while $\{$ exclude\} (optional) is the TaxID of the root of the excluded taxa in that subtree. Both TaxIDs are options of the script (a run with no TaxID related arguments will test the script).

\subsection{Using draftGenomes with Centrifuge and Recentrifuge}

Centrifuge's (Kim et al. 2016) nt+WGS databases are the result of pre-processing the NCBI BLAST nt database plus NCBI WGS sequences collected by draftGenomes so that they can be used by Centrifuge, thus allowing rapid and very sensitive classification within a huge range of organisms. Recentrifuge (Martí 2019) is able to post-process the results of Centrifuge (and several other classification engines) and provide an interactive, score-oriented visualization for the final outcome.

\section{Results}

In Martí et al. (2019), we prepared (December 2017) an ad hoc database for the Centrifuge classifier (Kim et al. 2016) including all the sequences from the nt database and some others from the NCBI WGS database obtained with draftGenomes. Those extra sequences belonged to the genus Olea and the kingdom Fungi. We used the resulting Centrifuge database to analyze several samples from a transcriptomic study of the evolution of Verticillium wilt of olive (Jimenez-Ruiz et al. 2017).

Figure 2 shows a comparison of results with the nt versus nt+WGS databases for the sample corresponding to olive tree roots 15 days after Verticilium dahliae inoculation. We see in the figure that, with the $n t+W G S$ database, the number of sequences classified passing the score filter was more than five times higher. The use of the nt database led to a final classification rate of $6.4 \%$ of the sequenced reads, whereas this rate increased to $34.7 \%$ when the $n t$ database was used together with the chosen sequences from the WGS database. We selected a high value (6o bp) as the threshold level for the score filter as recommended for RNA-sequencing projects (Kim et al. 2016). 
bioRxiv preprint doi: https://doi.org/10.1101/653592; this version posted June 4, 2019. The copyright holder for this preprint (which was not certified by peer review) is the author/funder, who has granted bioRxiv a license to display the preprint in perpetuity. It is made available under aCC-BY-NC-ND 4.0 International license.

\section{(A) Database: nt}

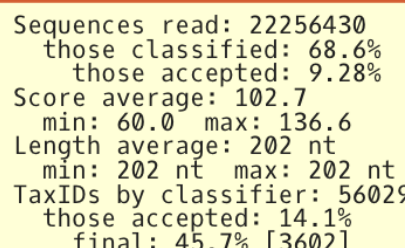

Sample statistics

Total sequences passing the score filter (60 bp): 1,418,315

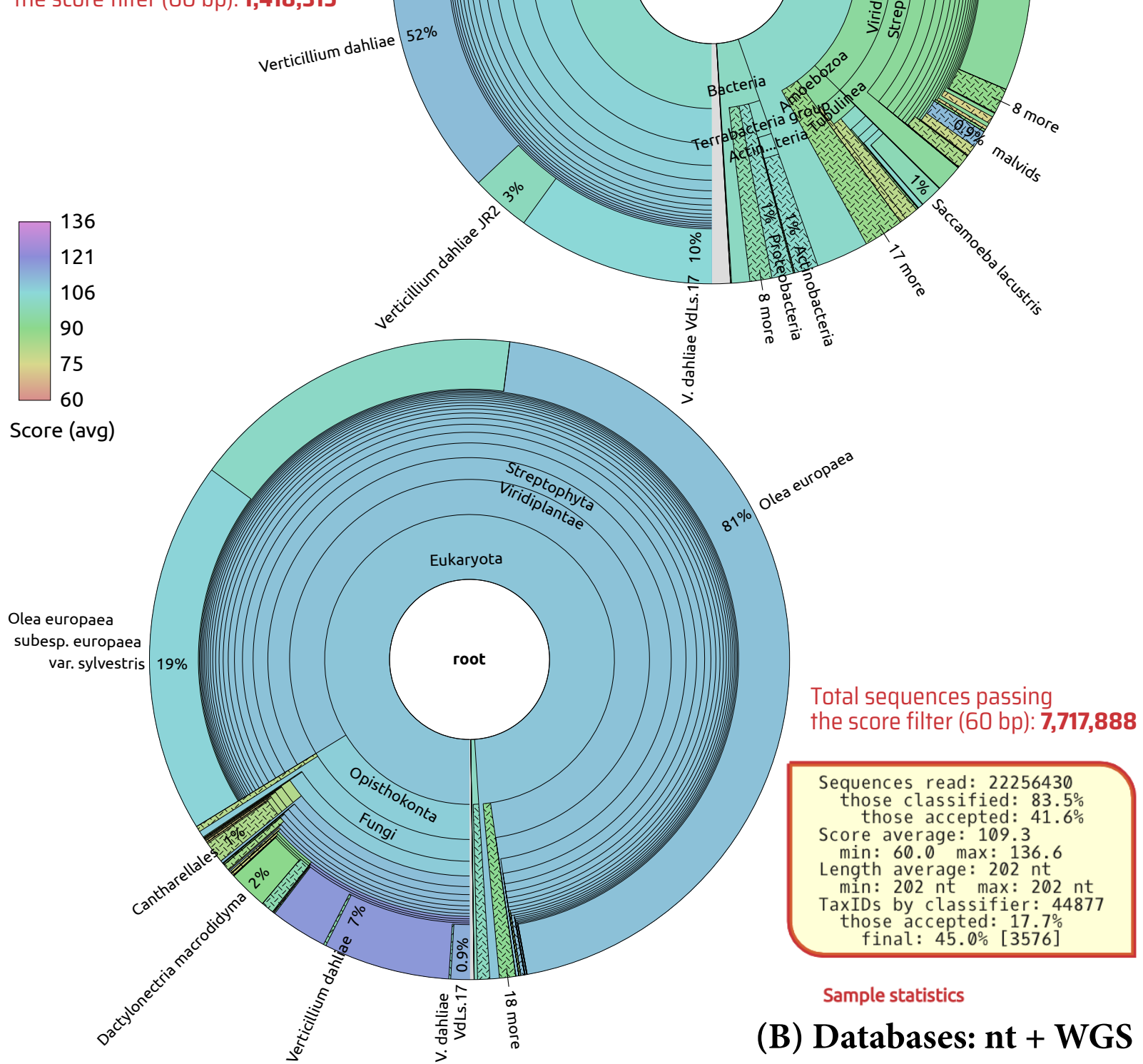

Figure 2. Comparison of results with the nt database versus sequences of WGS on top of the nt database. Recentr i fuge (Martí 2019) plots of SMS classified reads by Centrifuge, (A) using the nt database and (B) using the nt database plus all the sequences of the genus Olea and the kingdom Fungi present in the NCBI WGS database (Martí et al. 2019). The sample corresponds to Olive tree roots 15 days after Verticilium dahliae inoculation (Jimenez-Ruiz et al. 2017 ). The databases were retrieved and prepared in December 2017. 
Nevertheless, the increase in the classification rate was not at the expenses of a decrease in the confidence score. On the contrary, the average confidence score grew from 102.7 to 109.3. Interestingly, as Figure 2 also shows, the number of different NCBI taxonomic identifiers (TaxIDs field of the sample statistics) that were hit by the classifier also decreased with the addition of WGS data, especially those associated with low-quality scores. Therefore, the addition of draft genomes related to the host (the olive tree) not only markedly increased the number of previously unclassified reads that were finally classified as belonging to the host, but also reduced the number of previously misclassified reads, which ultimately were assigned to the olive tree as well. Specifically, the number of reads classified into the Olea europaea TaxID or below had a 31 -fold jump, and the score increased from 93.9 to 110.1 , thus indicating that not only one but both sequences of the paired-end reads were partially matching.

Although the main effect of adding WGS data affected the detection of the host, the addition of draft fungal genomes provided some improvements too. For example, Fusarium oxysporum, which is considered the pathogenic agent of Fusarium wilt of chickpea (García-Pedrajas et al. 1999), had a 27.6\% rise in the number of reads assigned and a $24.4 \%$ enhancement in the average confidence, to a relatively very high score of 120.5 .

\section{Discussion}

Although the idea of using draft genomes to improve the classification sensitivity is not new (Ames et al. 2015), we find in our studies (Martí et al. 2019; Bernabeu-Gimeno et al. 2018) that the WGS database is mature and rich enough to support a systematic use of its projects to supplement the nt database, thus minimizing the time gap between partial sequencing of new species and their identification in worldwide samples.

Finally, new algorithms for removing contaminants and low-complexity sequences from databases of draft genomes ( $\mathrm{Lu}$ and Salzberg 2018) strengthen our proposal. Our suggestion implies large customized databases reduced to the size of $\sim 100 \mathrm{~GB}$ using compression algorithms, currently feasible in fat computing nodes. The emergence of SCM (Storage Class Memory) will shortly democratize high-performance data analytics (Weiland et al. 2018), a new model that will allow huge metagenomic databases to be recreated with fresh WGS data in an ordinary computer, just before their use. 


\section{Availability}

The data and source code are anonymously and freely available on GitHub at https: //gi thub.com/ khyox/draftGenomes. The draftGenomes code is licensed under the GNU Affero General Public License Version 3 (https://uww.gnu.org/licenses/agpl.html). The readme file of the GitHub's repository is the most extensive and updated source of documentation for draftGenomes.

\section{Acknowledgments}

We would like to thank the SOM group (IFIC, Spain) for some high performance computing and storage resources that we used in our tests of draftGenomes.

\section{Author Contributions}

Conceptualization: JMM.

Data curation : JMM.

Formal analysis : JMM.

Investigation : JMM.

Methodology : JMM.

Project administration : JMM.

\section{Resources :}

Software : JMM.

Supervision : CPG.

Validation : JMM and CPG.

Visualization : JMM.

Writing - original draft : JMM.

Writing - review \& editing : JMM and CPG. 


\section{References}

Altschul SF, Gish W, Miller W, Myers EW, and Lipman DJ. 1990. Basic local alignment search tool. Journal of Molecular Biology. 21 5: 403-410.

Ames SK, Gardner SN, Marti JM, Slezak TR, Gokhale MB, and Allen JE. 2015. Using populations of human and microbial genomes for organism detection in metagenomes. Genome research. 25: 1056-1067.

Benson DA, Cavanaugh M, Clark K, Karsch-Mizrachi I, Lipman DJ, Ostell J, and Sayers EW. 2013. GenBank. Nucleic Acids Research. 41: D42.

Bernabeu-Gimeno M, Martí JM, Diaz-Villanueva W, Arnau V, and Peña-Garay C 2018. Gollum ecosystem: functional characterization of complex biological systems in extreme environments. In: XIV Symposium on Bioinformatics (JBI2018). Vol. 1. University of Granada, Genyo, BSC, and INB, p. 121.

Federhen S. 2011. The NCBI Taxonomy database. Nucleic Acids Research. 40: D143.

García-Pedrajas MD, Bainbridge BW, Heale JB, Pérez-Artés E, and Jiménez-Díaz RM. 1999. A Simple PCRbased Method for the Detection of the Chickpea-wilt Pathogen Fusarium oxysporum f.sp. ciceris in Artificial and Natural Soils. European Journal of Plant Pathology. 105: 251-259.

Jimenez-Ruiz J, O Leyva-Perez M de la, Schiliro E, Barroso JB, Bombarely A, Mueller L, Mercado-Blanco J, and Luque F. 2017. Transcriptomic Analysis of Olea europaea L. Roots during the Verticillium dahliae Early Infection Process. Plant Genome. 10:

Kim D, Song L, Breitwieser FP, and Salzberg SL. 2016. Centrifuge: rapid and sensitive classification of metagenomic sequences. Genome Research. 26: 1721-1729.

Lu J and Salzberg SL. 2018. Removing contaminants from databases of draft genomes. PLOS Computational Biology. 14: e1006277.

Martí JM. 2019. Recentrifuge: robust comparative analysis and contamination removal for metagenomics. PLOS Computational Biology. 15: e1006967.

Martí JM, Arias LF, Diaz-Villanueva W, Arnau V, Rodriguez-Franco A, and P. Garay C. 2019. Metagenomic dynamics in Olea europaea after root damage and Verticillium dahliae infection. bioRxiv. Dor: $1 \odot$. $1101 / 555185$.

Morgulis A, Gertz EM, Schäffer AA, and Agarwala R. 2006. A Fast and Symmetric DUST Implementation to Mask Low-Complexity DNA Sequences. Journal of Computational Biology. 13: 1028-1040. 
National Center for Biotechnology Information 2017. The BLAST databases README. Version 2017-03o6. National Library of Medicine (Bethesda, MD, USA). URL: ftp : / / ftp.ncbi .nlm.nih. gov/ blast/db/README.

NCBI Resource Coordinators. 2013. Database resources of the National Center for Biotechnology Information. Nucleic Acids Research. 41: D20.

Weiland M, Jackson A, Johnson N, and Parsons M. 2018. Exploiting the Performance Benefits of Storage Class Memory for HPC and HPDA Workflows. Supercomputing Frontiers and Innovations. 5:

Wheeler DL et al. 2007. Database resources of the National Center for Biotechnology Information. Nucleic acids research. 35: D12. 


\section{Supplemental Material of Not just BLAST nt: WGS database joins the party}

\section{Appendix A: Abbreviations}

BLAST Basic Local Alignment Search Tool

DDBJ DNA Data Bank of Japan

EMBL European Molecular Biology Laboratory

EST Expressed Sequence Tags NCBI database

GSS Genome Survey Sequences NCBI database

HTG High-throughput unfinished genome sequences NCBI database

NCBI National Center for Biotechnology Information

NIH National Institute of Health

nt Partially non-redundant nucleotide sequences NCBI database

PAT Patented sequences NCBI database

SCM Storage Class Memory

STS Sequence Tagged Sites NCBI database

WGS Whole-Genome Shotgun NCBI database 


\section{Appendix B: Step by step instructions on the use of draftGenomes in the gen- eration of a custom nt+WGS database for Centrifuge}

Currently, we need high-performance computing resources available to be able to generate the Centrifuge $n t+W G S$ kind of databases. Typically, a fat-node will do the job (we used, at least, a 32 cores machine with half a tebibyte memory and a fast scratch storage system). These are the detailed instructions, which assume that you have Centrifuge (Kim et al. 2016) already installed and available in your system:

1. Clone the draftGenomes GitHub repository, for example, under the user's home directory.

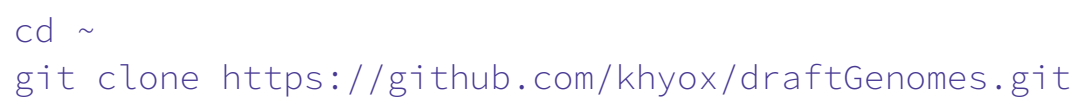

2. The following action is to download the NCBI $n t$ database and then to unzip it (both operations take some time as it has several tens of $\mathrm{GiB})$ :

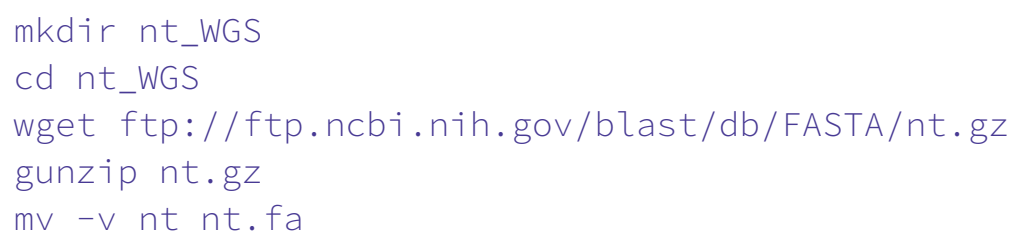

3. Proceed analogously with the taxdump databases (this is the shorter step):

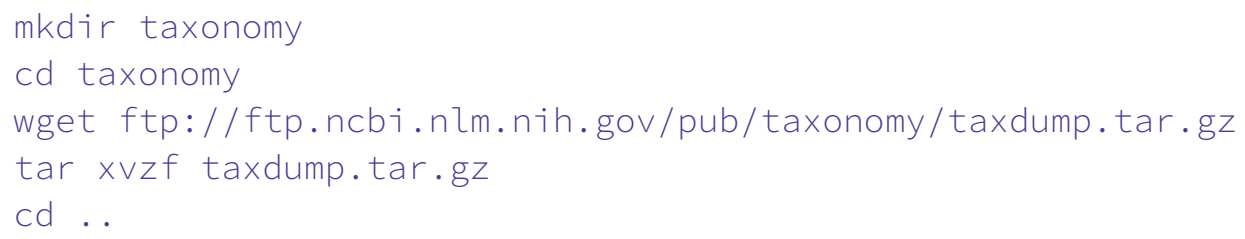

4. Use draftGenomes to automatically retrieve and consolidate the sequences of interest from the NCBI WGS database. For example, to obtain all the sequences associated with the genus Olea (NCBI TaxID 4145) and below, issue the following commands:

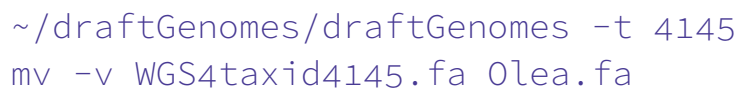

5. Repeat the previous step as needed if you would like draftGenomes to obtain different sets of sequences belonging to subtrees of unrelated TaxIDs (not intersecting, towards more specific levels of the taxonomic tree).

6. Now, to properly generate the accession to TaxID mapping file (that will be $\sim 10 \mathrm{GiB}$ ), the following commands are needed: 


\section{wget "ftp://ftp.ncbi.nih.gov/pub/taxonomy/accession2taxid/nucl_*.accession2taxid.gz" \\ wget ftp://ftp.ncbi.nih.gov/pub/taxonomy/accession2taxid/pdb. accession2taxid.gz \\ gunzip $-c *$.accession2taxid.gz | awk - $v$ OFS=' T' 'print \$2, \$3' > acc2tax.map}

7. This is an optional step that will mask low-complexity sequences by using DustMasker (Morgulis et al. 2006), a NCBI BLAST command-line application that should be installed in the system (with the rest of the NCBI tools or alone from here). The code dus tmasker should be executed with the DUST level (score threshold for subwindows) set to 20 , which is the default. Finally, all the masked nucleotides from the DustMasker output will be remasked as N using sed:

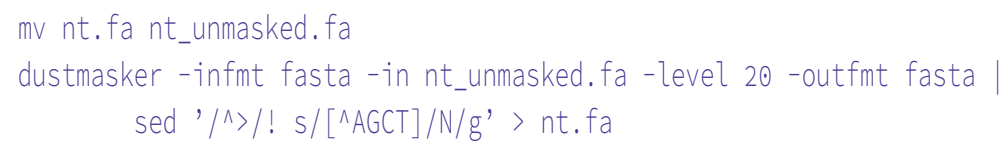

8. This is another optional step, and consists in repeating the last step but for any of the databases generated by draftGenomes. Just substitute the nt name for the applicable one, such as Olea for our example above.

9. Last but not least, the Centrifuge command that will generate the Centrifuge $n t+W G S$ database (this is the part that actually benefits from high performance computing) must be issued:

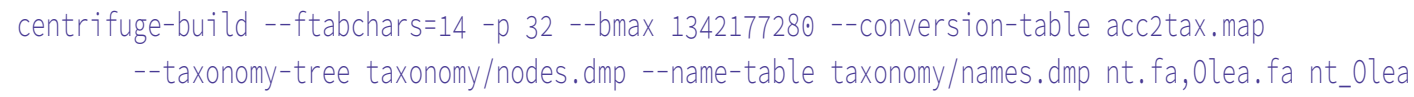

The last process finishes with a line like the following:

Total time for call to driver() for forward index: HH:MM:SS

That is the time of the last phase. In our case (using 32 cores) it took more than 20 hours. As this is not a short time, if centrifuge-bui ld is launched not using a batch system but in an interactive session, we strongly recommend using any mechanism to protect the process from unintentional interruptions, for instance by nohup:

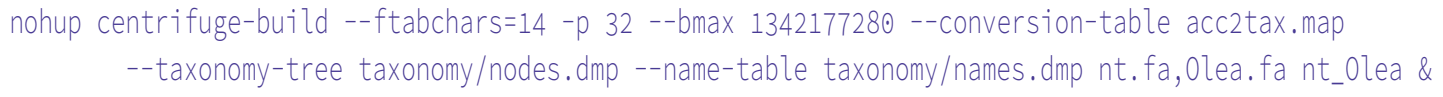

In this case, use tail -f nohup.out to safely follow the progress of the nt+WGS database build. 